\title{
Register rerum ad vol. 115
}

Originalarbeiten ohne Bezeichnung Original articles without asterisk Travaux originaux sans designation

Demonstrationen * Demonstrations * Demonstrations *

Sammelreferate **

Surveys **

Revues Générales **

Buchbesprechungen $* * *$ Book Reviews $* * *$ Livres Nouveaux $* * *$

Acanthose produite par acides et alcools

aliphatiques 35 Akanthosetest mit Teer und Teerkohlenwasserstoffen 374 Akne, berufliche (sog. Chlorakne), durch chlorierte aromatische zyklische Aether 540 Alopecie, terapia con raggi ultravioletti 743 Alopecie, toxische 749 Alpha Radiation from Polonium, Mode of Action of the, on Human Skin 661 Amyloidosis cutis nodularis atrophicans 508 Angiokeratoma corpis diffusum (Fabry)

572 Antimitotici, terapia antiblastica con 197 Atebrin, Wirkungsmechanismus 143 «Atopic Dermatitis», zum Begriff der

586

Bordet-Wassermann, auto-déviation du complement dans la reaction de 298

Bowen, Morbus, nach Zeckenstich 656

Bullöse Dermatosen, zur Diagnose des

«Zwei-Blasen-Symptoms» bei 547

Candida albicans, growth on keratin as

sole source of Nitrogen $81 \mathrm{C}$-Reactive protein in blood serum and

blister fluid in various dermatological

conditions 136 Cheilitis granulomatosa mit Fazialis-

parese und sekundärer Lingua plicata

761 Chlorakne, berufliche durch chlorierte

aromatische zyklische Äther 540 Chloroquin und Prednison, Behandlung

des Erythematodes 677 Chondroosteosarkom kombiniert mit

Basaliom $764 *$ Clorochina-Prednisone nel trattamento

dell'erythematodes 677 Congressus Internationalis Dermatolo-

gorum, Stockholm 1957838

Darier, Malattia di, ad insolita evolu-zione clinica 685

Dauerepilation mit Röntgenstrahlen 706

Dermatite atopique 223

Dermatite bulleuse mucosynéchante et atrophiante 205 
Dermatitis herpetiformis Duhring, Virus-ätiologie der 320

Dermatitis herpetiformis und Pemphigus, Inokulation von Blasenflüssig-keit auf Eihäute

bebrüteter Hühner-eier 452

Dermatochalasis $763 *$

Dermatomykosen $149 * *$

58 Dermatologica, Vol. 115, No. 6 (1957)

840

Register rerum ad vol. 115

Dermatomykosen 721

Dermatosen durch ektogene Ursachen

$60 * *$ Dermatosen durch endogene Ursachen

$69 * *$ Dermatologie, Gedanken zum Unter-

richt 186 Dermatose eruptive acro-située d'origine

probablement virosique 671 Dermogramme 482 Dosulfin-Exanthem $753 *$ Drescherkrankheit, ein Fall von 728 Dystrophie der Nagelplatte 428

Eczema due to turpentine, chemical

aspects 277 Eczema, experimental, effect of coal tar

and Hydrocortisone ointment on 272 Eczema experimental et tests cutanés

387 Eczema infantile 223 Ekzem durch Procain $755 *$ Ekzem, Photoallergisches (durch das Waschmittel Floris) $748 *$ Ekzem, Photoallergisches (durch Nadi-

san) $747 *$ Ekzem, «traumiteratives» (toxisches)

525 Elasticity of human skin related to age

106 Elephantiasis des membres inférieurs

534 Entzündungserregende Substanz der

Haut 502 Epidermodysplasia verruciformis 309 Epheliden $753 *$ Epidermodysplasia

verruciformis

(Lewandowsky-Lutz) 1 Epidermolysis bullosa hereditaria and

Porphyria cutanea tarda, differential

diagnosis 112 Epidermophytie, Zur Behandlung der

190 Epilation, dauernde, mit Röntgenstrah-

len 706 Erythema exsudativum multiforme bei

Panmyelopathie unbekannter Genese

$766 *$

Erythematodes, Behandlung mit Chlo-

roquin und Prednison 677 Excipient, $\Gamma$ influence de $\Gamma$, sur la résorp-

tion percutanée 530 Exanthem,Morbus-Kaposi-artiges 766 *

Fingerknöchelpolster $750 *$ Flogogene, sostanze le, della cute 502

Granuloma anulare $752 *$

Hautveränderungen durch ektogene Ursachen $60 * *$

Hautveränderungen durch endogene Ursachen $96 * *$

Herpes zoster generalisatus 758 *

Histopathologische Untersuchung, in der Dermatologie, zur Frage des Wer-tes der 516

Hydrocortisone ointment, the «thera-peutic» effect of 272

Hyperliprämie, idiopathische. Unter-suchungen über die Pathogenese der 579

Ichtyose congénitale 217 
Impetigo contagiosa, Beiträge eines

praktischen Dermatologen zur 406 Incontinentia pigmenti 623 Infektionskrankheiten 155

Jadassohn, Prof. W., Festschrift 165

Karzinoidose, intestinale, über Mund-schleimhautveränderungen bei malig-ner 641 Karzinom

auf Ulcus cruris $758 *$ Keloid nach Pockenimpfung $751 *$ Koebner's Phenomenon and

Pathogene-

sis of Psoriasis 51 Kräuselnaevus 119

Leprosy $780 * *$

Lichtreaktionen, Histologie der 345 Lipoid Proteinosis 23 Lungentuberkulose, Episoden aus der

Geschichte der Ätiologie der 304 Lupus érythémateux profond (Kaposi-

Irgang) 232

Register rerum ad vol. $115 \quad 841$

Lupus Vulgaris, Present Status of Vitamin D2 Treatment 491

Melanin Formation, Inhibition of 315 Melanomalignome an der Conjunctiva

bulbi, Röntgenweichstrahltherapie

von 633 Milien, eruptive, und Epithelioma ade-

noides cysticum Brooke 712 Mycoses, à propos de 721 Mykosen 149 **

Naevus sudoripare avec hyperidrose 293

Nékám, Lajos, Prof. Necrologia 80

Neurodermatitis, a Special Form of Allergy? 607

Neurodermitis disseminata, zum Begriíf der 586

Nickel Dermatitis, Spread of 596

Niederländischer Dermatologen-Verein, 149. Tagung am 26. Mai 1957 in Amsterdam 767-838

Nipple-test, La phosphatase alcaline dans la tétine de cobaye et le 181

Papulosis atrofizzante maligna di Degos

a decorso benigno 681 Pemphigoid, benign of mucous membrane 205 Pemphigus und Dermatitis

herpetifor-

mis, Inokulation von Blasenflüssig-

keit auf Eihäute bebrüteter Hühner-

eier 452 Pemphigus oculaire 205 Pemphigus, Über die Virusätiologie des

320 Pemphigus vulgaris 756 * Phosphatase alcaline, la, dans la tétine

de cobaye et le «Nipple-test» 181 Plasmalreaktion, histochemische Dar-stellung der, an gesunder und kranker

Haut 444 Porphyria cutanea tarda and Epidermo-

lysis buUosa hereditaria, differential

diagnosis 112 Pringle, malattia di, Sulle alterazioni

mucose nella 333

Protein, C-Reactive in blood serum and

blister fluid in various dermatological

conditions 136 Prurigo de Besnier 223 Pseudoxanthoma elasticum 382 Psoriasis-Arthritis 467

Psoriasis pustulosa, Rhythmisch verlau-

fende Eruptionen bei 616 Psoriasis, Pathogenesis of 51 Pterygiumsyndrom Typ Bonnevie-UU-

rich mit Erythrokeratosis palmaris et

plantaris $759 *$ Pyoderma gangraenosum mit Lungen-

veränderungen 648

Recklinghausen, malattia di, Tratta- 
mento chirurgico di un caso di, con

imponente «tumore reale» 693 Résorption percutanée, Influence del'ex-

cipient sur la 530 Röntgenstrahlen, Dauerepilation mit

706 Röntgenweichstrahltherapie von Mela-

nomalignomen an der Conjunctiva

bulbi 633 Ruiter-Pompen, Syndrome cardio-vaso-

rénal de 572

Sclérodermie progressive, les lesions oesophagiennes dans la 700

Senear-Usher Syndrom 756 *

Société Suisse de Dermatologie et de Vénéréologie Colloquium Dermatolo-gicum, Bellinzona, le 12 mai 1957, I. Communications 671-747, II. Demonstrations 747-766

Sulfamidici, Manifestazioni ulcero-ne-crotiche della cute da, intolleranza topica ai 697

Syphilis $809 * *$

Syphilis, neue Nachuntersuchung des «Boeck-Bruusgaard'schen Materials » 476

Syphilis, endemic, Penicillin treatment of 287

Syphilis, non-veneral «Endemic», epi-demiological aspects of 248

842

Register rerum ad vol. 115

Schweizerische Gesellschaft für Derma-tologie und Venereologie. Colloquium Dermatologicum, Bellinzona, den 12. Mai 1957, I. Mitteilungen Seite 671-747, II. Demonstrationen Seite 747-766 Sto $\Lambda \Lambda$ vechseldermatosen, «fakultative», Erfahrungen mit der temporären Keimverarmung des Darmes bei 459

Strahlenbiologie und -therapie $824 * *$

Strahlenreaktionen, Histologie der licht-bedingten 345

Strahlentherapie, Dauerepilation mit Röntgenstrahlen 706

Strahlentherapie, Distanzbestrahlung 689

Striae distensae $763 *$

Sturge-Weber-Krabbe'sche Krankheit, Klinischer und pathologisch-anato-mischer Beitrag zur 555

Telepanirradiazione di dermatosi gene-ralizzate coi tubi a finestra di Berillio 689

Tests épicutanés et eczema experimental 387

Tinea Tonsurans of the Scalp, Morphological Alterations in Parasitized

Hairs Observed in the Course of Topical Treatment of 496

Trebbiatori, un caso di malattia dei 728

Trichomalacie, contribution à Гétiologie de la 717

Trichophyton epilans, Infections of fifty years duration in two sisters 40

Tuberkulose der Haut $774 * *$

Ultraschall in der Dermatologie, Erfahrungen mit 121

Ultraviolette Strahlen, Therapie der Alopecie mit 743

Urticaria Solaris 171

UV - Reaktion an der menschlichen Haut $838 * * *$

Venose Dekompensation, Probleme der

732

Whipple, maladie de, Réticulo-endothé-liose cutanée dans la 358 
X-ray therapy in dermatology, factors influencing the quantity of radiation reaching the gonadal areas 433

«Zwei-Blasen-Symptom», zur Diagnose des, bei bullösen Dermatosen 547 